\title{
Switching On/off of Electroactivity of Hemin/guanine-quadruplex Complex as DNA Aptazyme Triggered by Response to Histamine
}

\author{
Hiroshi Aoki* and Tetsuya Nakazato \\ Environmental Management Research Institute, \\ National Institute of Advanced Industrial Science and Technology (AIST), \\ 16-1 Onogawa, Tsukuba, Ibaraki 305-8569, Japan
}

(Received November 12, 2019; accepted January 27, 2020)

Keywords: switching probe, switch on/off, DNA aptamer, aptazyme, hemin, G-quadruplex, histamine

We newly developed a histamine-triggered electrochemical switching aptazyme by utilizing a hemin molecule and a hemin-targeting aptamer (AptDNA) complex (hemin/AptDNA). We spectroscopically and electrochemically found that the electroactivity of hemin/AptDNA is suppressed or restored in the presence or absence of histamine, respectively. The observed hyperchromicity of the Soret band of hemin at $404 \mathrm{~nm}$ showed the interaction of hemin with the G-quadruplex structure of AptDNA. The absorbance at $418 \mathrm{~nm}$ for the hemin/AptDNA solution increased in the presence of dihydrogen peroxide $\left(\mathrm{H}_{2} \mathrm{O}_{2}\right)$ and 2,2'-azinobis(3ethylbenzothiozoline)-6-sulfonic acid (ABTS), where the colorless $\mathrm{ABTS}^{2-}$ was oxidized to the green $\mathrm{ABTS}^{\circ-}$. These results led us to conclude that hemin/AptDNA worked as a redox aptazyme for the redox reaction of $\mathrm{H}_{2} \mathrm{O}_{2}$ and ABTS. The presence of histamine inhibited this reaction. This was also electrochemically ascertained. Electrochemical investigation demonstrated that the reduction reaction of $\mathrm{H}_{2} \mathrm{O}_{2}$ was facilitated by hemin/AptDNA compared with the case of AptDNA and that the reaction was restrained by the addition of histamine to the solution. These spectroscopic and electrochemical investigations revealed that the electroactivity of hemin/AptDNA is switched on/off in the presence/absence of histamine as a redox aptazyme, respectively.

\section{Introduction}

In the last few decades, oligonucleotide detection was investigated as indicators of chemical toxicity and for biomedical diagnosis. For testing chemical toxicity, cell-based assays are widely performed using model cells exposed to chemicals and are based on oligonucleotide detection techniques for DNAs and RNAs reflecting biological responses to chemical stimuli. ${ }^{(1,2)}$ Among biomedical diagnoses, cancer diagnosis is one of the attractive applications of oligonucleotide detection, where micro-RNAs (miRNAs), emerging in the past decades, are present in the blood of cancer patients and can be utilized as cancer biomarkers specific to the types and stages of cancers. $^{(3,4)}$

*Corresponding author: e-mail: aoki-h@aist.go.jp

https://doi.org/10.18494/SAM.2020.2705 
For faster and simpler detection, a great deal of research has been carried out for the development of oligonucleotide detection techniques based on various approaches, including microgravimetric, optical, and electrochemical techniques. ${ }^{(5)}$ In particular, electrochemical techniques have remarkable merits in terms of the simple design and size reduction of detection systems. ${ }^{(6)}$

We have studied electrochemical oligonucleotide detection sensing techniques, without labeling target oligonucleotides, aiming at the simple and rapid detection of target oligonucleotides. Recently, we have developed "switching probe molecules", i.e., artificially synthesized probe molecules with redox active moieties switching electrochemical signals on or off upon hybridization with target oligonucleotides. ${ }^{(7)}$ One is the probe with a ferrocene moiety $(\mathrm{Fc})$ at the probe terminal. ${ }^{(8,9)}$ Before hybridization, the flexible single-stranded (ss) probe structure allows the terminal $\mathrm{Fc}$ to access the electrode surface and yield the redox current for Fc redox reaction. After hybridization, in contrast, the formation of a double-stranded (ds) probe/target pair inhibits the access and decreases the redox current. The other one is the probe with $\mathrm{Fc}$ as an electroactive moiety at one terminal and $\beta$-cyclodextrin $(\beta-C D)$ as its suppresseor moiety at the other terminal. ${ }^{(10)}$ Before hybridization, the formation of an internal complex between $\mathrm{Fc}$ and $\beta$-CD suppresses the redox activity of Fc. After hybridization, in contrast, the formation of the ds-probe/target pair makes the $\mathrm{Fc} / \beta-\mathrm{CD}$ complex dissociate and allows the redox activity of $\mathrm{Fc}$ to be restored. These switching probe molecules have electroactive moieties that can generate electrochemical signals sensitive to hybridization with target oligonucleotides. Therefore, they have the advantage of omitting the use of external electroactive markers. This advantage can greatly contribute to the simple and rapid detection of oligonucleotides.

For further development of the switching probes to enhance the detection signals, we studied the usage of redox enzymes in the switching system. Redox enzymes are popularly used to enhance the electrochemical signals as catalysts where they conduct multiple-electron reactions, ${ }^{(11,12)}$ whereas redox molecules, such as Fc used in our previous studies, conduct oneelectron reactions.

Recently, redox enzymes based on hemin-involving enzymatic DNA aptamers (aptazymes) have been studied. ${ }^{(13-15)}$ The aptazymes are composed of a hemin, an Fe-coordinated porphyrin, and a hemin-targeting aptamer (AptDNA). The aptamer forms a stable guanine $(\mathrm{G})$-quadruplex structure, and a hemin is intermolecularly bound to the G-quadruplex structure, as schematically shown in Fig. 1. ${ }^{(16)}$ Aptazymes are artificial enzymes, which are considered more favorable for the fabrication of sensors from the viewpoint of sensor lifetimes than naturally occurring enzymes. Moreover, they are easily synthesized via DNA synthesizers. There are many reports on the bioanalytical applications of aptazymes based on various approaches, ${ }^{(17)}$ including electrochemical, ${ }^{(18)}$ optical (colorimetric, fluorescent) ${ }^{(19)}$ and chemiluminescent ${ }^{(20)}$ approaches. To the best of our knowledge, nevertheless, the targetsensitive switching function of the electroactivity of the hemin/G-quadruplex complex as an aptazyme has rarely been studied. Therefore, in this study, we performed basic investigations of the multiplex-electron-reaction-based switching function of the hemin/G-quadruplex complex triggered by molecular recognition as an aptazyme that is the signal generation moiety for switching probes. 

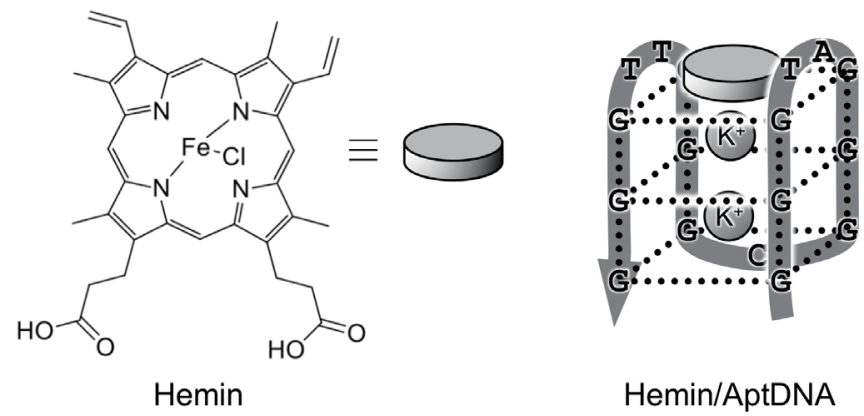

Fig. 1 Chemical structure of hemin and structure of hemin/AptDNA. The structure is stabilized by a $\mathrm{K}^{+}$cation.

In this study, we developed a novel histamine-triggered electrochemical switching aptazyme by utilizing a hemin molecule and an AptDNA complex (hemin/AptDNA). It is known that an imidazole moiety coordinates to a central metal Fe of hemin. ${ }^{(21,22)}$ We expected that the binding of histamine, having an imidazole moiety, would modulate the switching of the aptazyme activity. We spectroscopically and electrochemically found that the electroactivity of hemin/AptDNA is suppressed or restored in the presence or absence of histamine, respectively. The observed hyperchromicity of the Soret band of hemin at $404 \mathrm{~nm}$ showed the binding of hemin to the G-quadruplex structure of AptDNA. The absorbance at $418 \mathrm{~nm}$ for the hemin/ AptDNA solution increased in the presence of dihydrogen peroxide $\left(\mathrm{H}_{2} \mathrm{O}_{2}\right)$ and 2,2'-azinobis(3ethylbenzothiozoline)-6-sulfonic acid (ABTS), where the colorless $\mathrm{ABTS}^{2-}$ was oxidized to the green $\mathrm{ABTS}^{-}{ }^{-}$. These results led us to conclude that hemin/AptDNA worked as a redox aptazyme for the redox reaction of $\mathrm{H}_{2} \mathrm{O}_{2}$ and ABTS. The presence of histamine inhibited this reaction. This was also electrochemically ascertained. Electrochemical investigation demonstrated that the reduction reaction of $\mathrm{H}_{2} \mathrm{O}_{2}$ was facilitated by hemin/AptDNA compared with the case of AptDNA and that the reaction was restrained by the addition of histamine to the solution. These spectroscopic and electrochemical investigations revealed that the electroactivity of hemin/AptDNA is switched on/off in the absence/presence of histamine as a redox aptazyme, respectively.

\section{Materials and Methods}

\subsection{Reagents}

The synthesized oligonucleotide was purchased from Eurofins Genomics Japan (Tokyo, Japan), the sequence of which was 5' GGG TAG GGC GGG TTG GG 3' (AptDNA). Sodium dihydrogen phosphate $\left(\mathrm{H}_{2} \mathrm{NaPO}_{3}\right)$, sodium hydroxide $(\mathrm{NaOH})$, potassium hydroxide $(\mathrm{KOH})$, potassium chloride $(\mathrm{KCl})$, dihydrogen sulfate $\left(\mathrm{H}_{2} \mathrm{SO}_{4}\right), \mathrm{H}_{2} \mathrm{O}_{2}$, ABTS, hemin, and histamine were purchased from Wako Chemical (Tokyo, Japan). 10X TE buffer (100 mM Tris-HCl buffer, $10 \mathrm{mM}$ EDTA, $\mathrm{pH}$ 8.0; Tris and EDTA represent tris(hydroxymethyl)aminomethane and ethylenediamine-tetraacetic acid, respectively) was from Dojindo (Kumamoto, Japan). Triton X-100 (octylphenol polyethoxylate) was from Sigma-Aldrich (Tokyo, Japan). All of the 
chemicals used were of analytical reagent grade. All aqueous solutions were prepared with deionized and charcoal-treated water (specified resistance > 18.2 M 2 ), obtained using a Milli-Q reagent-grade water system (Millipore; Bedford, MA). In this study, buffer solutions containing the $\mathrm{K}^{+}$cation were used. This is because the $\mathrm{K}^{+}$cation stabilizes the G-quadruplex structure (Fig. 1), four guanines of which are connected to each other by Hoogsteen hydrogen bonds. ${ }^{(16)}$

\subsection{Spectroscopic measurement}

Spectroscopic measurement was performed using a NanoDrop 2000c UV-vis spectrometer (Thermo-Fisher; Tokyo, Japan) in a 20 -fold-diluted 10X TE buffer (5 mM Tris-HCl buffer, $0.5 \mathrm{mM}$ EDTA) $+10 \mathrm{mM} \mathrm{KCl}+0.02 \%$ Triton X-100. The concentrations of AptDNA were also measured using a NanoDrop 2000c. A solution of AptDNA was prepared as follow. The TE buffer containing $50 \mu \mathrm{M}$ AptDNA was incubated at $95{ }^{\circ} \mathrm{C}$ for $5 \mathrm{~min}$ and then gradually cooled to $25{ }^{\circ} \mathrm{C}$ at a rate of $1{ }^{\circ} \mathrm{C} \mathrm{min}{ }^{-1}$ to form a G-quadruplex structure, for the preparation of an AptDNA solution. A hemin solution is the TE buffer containing $50 \mu \mathrm{M}$ hemin. A hemin/AptDNA solution is the TE buffer containing $50 \mu \mathrm{M}$ AptDNA and $50 \mu \mathrm{M}$ hemin. The temperature of the solutions was controlled using a Thermal Cycler Dice Gradient TP600 (TAKARA; Tokyo, Japan).

\subsection{Colorimetric analyses of hemin/AptDNA}

Colorimetric analyses of hemin/AptDNA solutions were performed by visually comparing the colors of the hemin/AptDNA solutions containing $1 \mathrm{mM}$ ABTS, $1 \mathrm{mM} \mathrm{H}_{2} \mathrm{O}_{2}, 1 \mathrm{mM}$ ABTS $+1 \mathrm{mM} \mathrm{H}_{2} \mathrm{O}_{2}$, or $1 \mathrm{mM} \mathrm{ABTS}+1 \mathrm{mM} \mathrm{H}_{2} \mathrm{O}_{2}+1 \mathrm{mM}$ histamine. The color changes were qualitatively expressed from "--" (as negative) to "+" (as positive).

\subsection{Preparation of gold disk electrodes}

Gold disk electrodes $\left(2 \mathrm{~mm}^{2}\right.$ area) were purchased from Bioanalytical Systems (BAS) (Tokyo, Japan) and used in all the electrochemical experiments. The electrodes were prepared according to previous reports. ${ }^{(8,23,24)}$ Briefly, the electrodes were mechanically polished with wet 0.3 and $0.05 \mu \mathrm{m}$ alumina slurries (Buehler; Lake Bluff, IL). The polished electrodes were electrochemically polished by cycling the potential between -0.4 and $-1.2 \mathrm{~V}$ in $0.5 \mathrm{M} \mathrm{KOH}$ solution until the cyclic voltammogram $(\mathrm{CV})$ did not change. Then, the electrode was dipped in $1 \mathrm{M} \mathrm{H}_{2} \mathrm{SO}_{4}$ solution, followed by cycling the potential between 0 and $+1.6 \mathrm{~V}$ until a constant $\mathrm{CV}$ was observed.

\subsection{Electrochemical measurement}

Electrochemical measurement was performed at room temperature with a three-electrode configuration consisting of a $\mathrm{Ag} / \mathrm{AgCl}$ reference electrode, a platinum auxiliary electrode, and the prepared gold disk electrode as a working electrode, using an ALS 760C electrochemical 
analyzer (BAS). Cyclic voltammetry was performed in $5 \mathrm{mM}$ phosphate-buffered solution $\left(\mathrm{pH} 7.0, \mathrm{Na}^{+}\right.$salt $)+20 \mathrm{mM} \mathrm{KCl}$. The potential at the working electrode was scanned from +0.2 to -1.0 and back to $+0.2 \mathrm{~V}$ at a rate of $0.1 \mathrm{~V} \mathrm{~s}^{-1}$. CVs were measured for $50 \mu \mathrm{M}$ AptDNA, $50 \mu \mathrm{M}$ AptDNA $+0.5 \mathrm{mM}$ hemin (hemin/AptDNA), $50 \mu \mathrm{M}$ AptDNA $+0.5 \mathrm{mM}$ hemin + $50 \mu \mathrm{M}$ histamine (hemin/AptDNA + histamine), and a bare gold electrode in phosphatebuffered saline (PBS), PBS containing $0.6 \mathrm{mM} \mathrm{H}_{2} \mathrm{O}_{2}$, and PBS containing $5 \mathrm{mM} \mathrm{H}_{2} \mathrm{O}_{2}$. All electrochemical solutions were prepared from PBS deoxygenated by purging with Ar gas for $15 \mathrm{~min}$.

\section{Results and Discussion}

\subsection{Spectroscopic characterization of hemin/AptDNA}

To ascertain the interaction of hemin with the G-quadruplex structure of AptDNA to form hemin/AptDNA and to study the function of hemin/AptDNA as a redox enzyme, the spectroscopic characterization of hemin/AptDNA was firstly performed. Figure 2 shows spectra for AptDNA, hemin, and hemin/AptDNA. The spectrum for AptDNA (dotted line) showed a large absorption peak at around $260 \mathrm{~nm}$, corresponding to the presence of nucleotides. The spectrum for hemin showed no remarkable peaks except for a broad and small peak at around $395 \mathrm{~nm}$ (dashed line), corresponding to the Soret band of hemin. Next, the hemin/AptDNA prepared by the incubation of hemin with AptDNA gradually cooled from 95 to $25{ }^{\circ} \mathrm{C}$ for 60 min showed an markedly large absorption peak at $406 \mathrm{~nm}$, slightly redshifted from $395 \mathrm{~nm}$. This sharp hyperchromicity of the Soret band of hemin suggests the interaction of the porphyrin moiety of hemin with the G-quadruplex structure of AptDNA (Fig. 1). This consequently caused the increase in the planarity of the hemin porphyrin ring and the expansion of the hemin $\pi$-conjugated system.

In this study, we used Triton X-100 to increase the solubility of hemin to aqueous solutions. However, Triton X-100 has large absorption peaks at around $270 \mathrm{~nm}$ derived from an aromatic moiety. ${ }^{(25)}$ Therefore, Triton X-100 was used as minimally as possible so as not to affect the

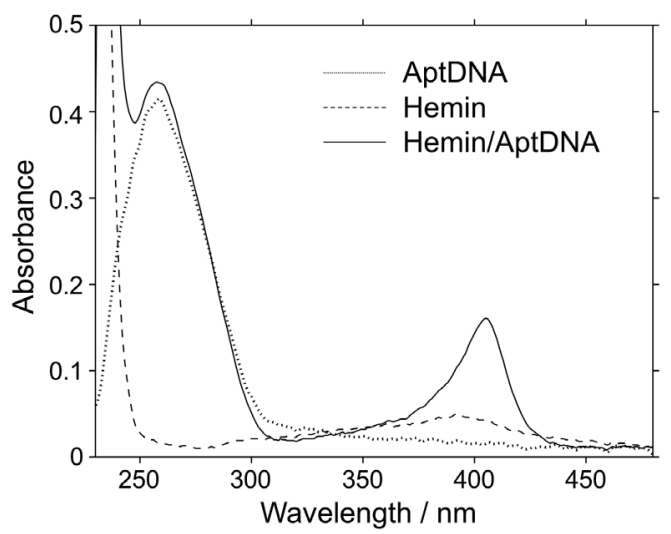

Fig. 2. UV-vis spectrum of PBS solutions containing AptDNA (dotted line), hemin (dashed line), and hemin/AptDNA (solid line). 
measurements. In Fig. 2, the spectrum for hemin shows no absorption peaks caused by the aromatic moiety, and we considered that there was no effect of Triton X-100 on the study.

\subsection{Colorimetric characterization of hemin/AptDNA}

To further investigate the function of the thus prepared hemin/AptDNA as a redox enzyme, we performed the colorimetric analysis of the redox activity of the complex to ABTS and $\mathrm{H}_{2} \mathrm{O}_{2}$. We added only ABTS, $\mathrm{H}_{2} \mathrm{O}_{2}$, or both ABTS and $\mathrm{H}_{2} \mathrm{O}_{2}$ to the solutions containing hemin/ AptDNA (Table 1). In the only case of ABTS and $\mathrm{H}_{2} \mathrm{O}_{2}$, the solution showed the change in color from colorless to green and a large and sharp absorbance at $418 \mathrm{~nm}$ [Table 1(D)]. This phenomenon is due to the redox reaction of ABTS and $\mathrm{H}_{2} \mathrm{O}_{2}$ via hemin/AptDNA as a redox mediator, as follows.

$$
2 \mathrm{ABTS}^{2-} \text { (colorless) }+\mathrm{H}_{2} \mathrm{O}_{2}+2 \mathrm{H}^{+} \rightleftarrows 2 \mathrm{ABTS}^{\bullet-} \text { (green) }+2 \mathrm{H}_{2} \mathrm{O}
$$

In contrast, the addition of histamine to the solution showed only a relatively small change in color [Table 1(E)]. This indicates that histamine restrains the above reaction in Eq. (1).

These results tell us that hemin/AptDNA induced from the interaction of hemin with the G-quadruplex structure of AptDNA mediates the redox reaction between ABTS and $\mathrm{H}_{2} \mathrm{O}_{2}$ (Fig. 3 , upper), and it also tell us that the presence of histamine in the solution restrains the redox

Table 1

Colorimetric analysis of hemin/AptDNA solutions with $1 \mathrm{mM} \mathrm{ABTS}, 1 \mathrm{mM} \mathrm{H} \mathrm{O}_{2}$, and/or $1 \mathrm{mM}$ histamine. Solutions without any components (A), with only ABTS (B), with only $\mathrm{H}_{2} \mathrm{O}_{2}$ (C), with ABTS and $\mathrm{H}_{2} \mathrm{O}_{2}$ (D), and with $\mathrm{ABTS}, \mathrm{H}_{2} \mathrm{O}_{2}$, and histamine (E). The color changes are qualitatively expressed from "--" (as negative) to "+" (as positive).

\begin{tabular}{lccccc}
\hline & $(\mathrm{A})$ & $(\mathrm{B})$ & $(\mathrm{C})$ & $(\mathrm{D})$ & $(\mathrm{E})$ \\
\hline $\mathrm{ABTS}$ & - & + & - & + & + \\
$\mathrm{H}_{2} \mathrm{O}_{2}$ & - & - & + & + & + \\
Histamine & - & - & - & - & + \\
\hline Color change & - & - & - & +++ & + \\
\hline
\end{tabular}
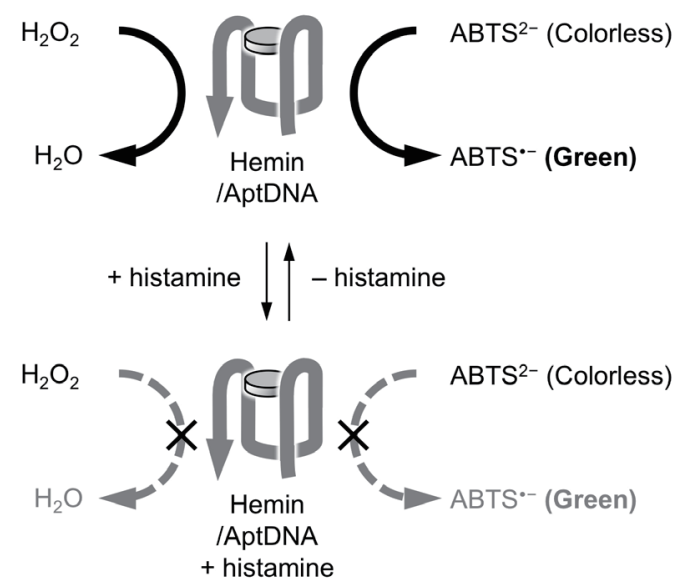

Fig. 3. Working principle of hemin/AptDNA as an aptazyme mediating a redox reaction of $\mathrm{ABTS}^{2-}$ (colorless) and $\mathrm{H}_{2} \mathrm{O}_{2}$ to $\mathrm{ABTS}^{--}$(green) and $\mathrm{H}_{2} \mathrm{O}$. The reaction is inhibited in the presence of histamine and restored in the absence of histamine. 
reaction (Fig. 3, lower). As a result, the prepared hemin/AptDNA worked as a redox enzyme that can switch on or off its own activity as a mediator in the absence or presence of histamine, respectively.

\subsection{Electrochemical characterization of hemin/AptDNA}

Next, the electrochemical characterization of hemin/AptDNA was performed to demonstrate its electrochemical function as a switching aptazyme responding to the presence of histamine. CVs were measured in solutions of AptDNA, hemin/AptDNA, and hemin/AptDNA + histamine containing $0 \mathrm{M}, 0.6 \mathrm{mM}$, and $5 \mathrm{mM} \mathrm{H}_{2} \mathrm{O}_{2}$ (Fig. 4).

Figure 4(a) shows the CVs measured in the solutions containing $0 \mathrm{M} \mathrm{H}_{2} \mathrm{O}_{2}$. For AtpDNA, there were no redox peaks in the $\mathrm{CV}$, but for hemin/AptDNA, small and broad peaks were observed at -0.293 and $-0.074 \mathrm{~V}$. The latter peak can be attributed to the reduction reaction of hemin in the presence of residual $\mathrm{O}_{2}$ in the solution. ${ }^{(26)}$ Figure $4(\mathrm{~b})$ shows the CVs measured in the solutions containing $0.6 \mathrm{mM} \mathrm{H}_{2} \mathrm{O}_{2}$. For AtpDNA, a large peak at $-0.687 \mathrm{~V}$ and a relatively

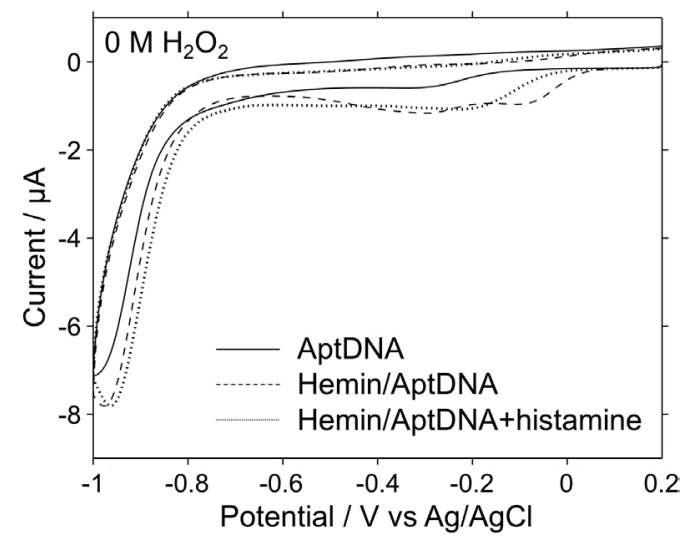

(a)

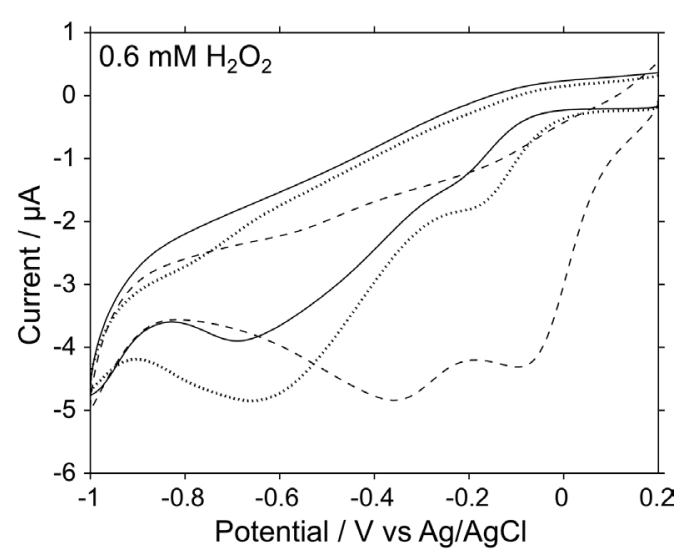

(b)

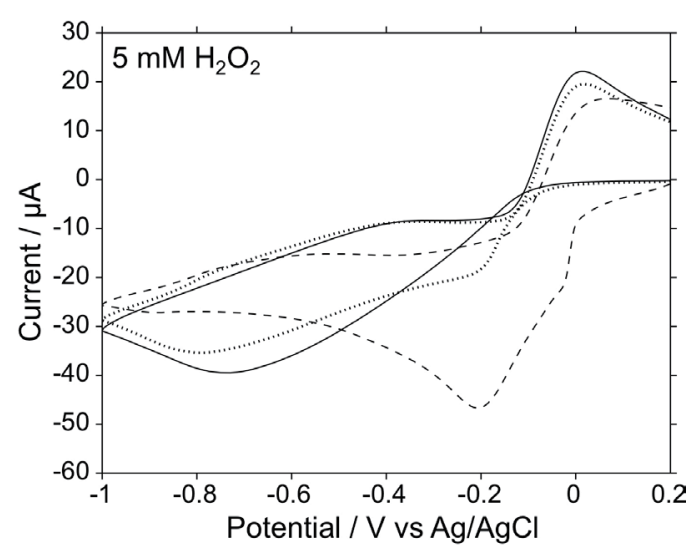

(c)

Fig. 4. CVs for electrochemistry of AptDNA (solid line), hemin/AptDNA (dashed line), and hemin/AptDNA + histamine (dotted line) in PBS containing (a) $0 \mathrm{M}$, (b) $0.6 \mathrm{mM}$, and (c) $5 \mathrm{mM} \mathrm{H}_{2} \mathrm{O}_{2}$. 
small peak at $-0.219 \mathrm{~V}$ were observed, showing the reduction reaction $\mathrm{H}_{2} \mathrm{O}_{2}$. On the other hand, for hemin/AptDNA, two large reduction peaks were observed at -0.349 and $-0.091 \mathrm{~V}$. The large and positive shift of these peaks indicates that the reduction reaction of $\mathrm{H}_{2} \mathrm{O}_{2}$ was facilitated by hemin/AptDNA. Moreover, the addition of histamine in the solution decreased the reduction peaks of $\mathrm{H}_{2} \mathrm{O}_{2}$ and the potentials were restored to $-0.657 \mathrm{~V}$ (a large peak) and $-0.184 \mathrm{~V}$ (a small peak), similarly to the case of AptDNA.

Furthermore, this tendency was markedly observed in higher $\mathrm{H}_{2} \mathrm{O}_{2}$ concentrations. Figure 4(c) shows the CVs measured in the solutions containing $5 \mathrm{mM} \mathrm{H}_{2} \mathrm{O}_{2}$. For AptDNA, a reduction reaction for $\mathrm{H}_{2} \mathrm{O}_{2}$ was observed as a broad peak at $-0.730 \mathrm{~V}$. For hemin/AptDNA, however, the reaction was observed as a large and sharp peak at $-0.202 \mathrm{~V}$ accompanied by a small peak at $-0.025 \mathrm{~V}$. In contrast, after the addition of histamine to the solution, the reaction peak was observed at $-0.800 \mathrm{~V}$, which weakened and shifted to the original potential shown as in the case of AptDNA.

Considering these results, it can be concluded that $\mathrm{H}_{2} \mathrm{O}_{2}$ is reduced via hemin/AptDNA as a mediator (Fig. 5, upper) and that the presence of histamine restrained the reduction reaction of $\mathrm{H}_{2} \mathrm{O}_{2}$ (Fig. 5, lower). Here, the working principle of the switching mechanism of the hemin/AptDNA aptazyme responding to histamine is still unknown. We consider that this is partially based on the coordination of an imidazole moiety of histamine to a central metal Fe of hemin. ${ }^{(21)}$ The coordination of histamine can affect the reduction reaction of $\mathrm{H}_{2} \mathrm{O}_{2}$. For more detailed information on the switching mechanism, further studies should be carried out.

\subsection{Electrochemical signal switching of hemin/AptDNA in the absence and presence of histamine}

In Sect. 3.3, on the basis of the difference between the measured CVs of $\mathrm{H}_{2} \mathrm{O}_{2}$, we mentioned that the reduction reaction of $\mathrm{H}_{2} \mathrm{O}_{2}$ was facilitated by hemin/AptDNA and that the reaction

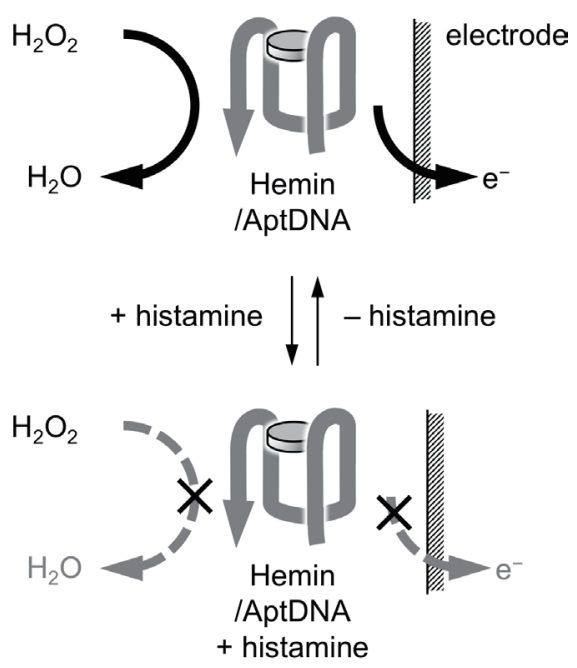

Fig. 5. Working principle of hemin/AptDNA as an aptazyme mediating a reductive reaction of $\mathrm{H}_{2} \mathrm{O}_{2}$ to $\mathrm{H}_{2} \mathrm{O}$ on an electrode surface. The reaction is inhibited in the presence of histamine and restored in the absence of histamine. 
was restrained by the addition of histamine. The peak potentials of $\mathrm{H}_{2} \mathrm{O}_{2}$ reduction markedly changed here in the measurement. By focusing on the current change at a fixed potential, we expect to more easily comprehend the switching effect of hemin/AptDNA.

In Fig. 4(b), the CV for hemin/AptDNA shows the reduction current at the reduction peak potential $(=-0.349 \mathrm{~V})$ of $-4.838 \mu \mathrm{A}$, whereas those for AptDNA and Hemin/AptDNA + histamine show -2.063 and $-2.469 \mu \mathrm{A}$, respectively. Namely, the reduction current increased about twofold (from AptDNA to hemin/AptDNA) and decreased about 1/2-fold (from hemin/AptDNA to hemin/AptDNA + histamine). Figure 4(c) shows more remarkable changes. The CVs for hemin/AptDNA show the reduction current at the reduction peak potential $(=-0.202 \mathrm{~V})$ of $-46.63 \mu \mathrm{A}$, whereas those for AptDNA and Hemin/AptDNA + histamine show -10.03 and $-18.44 \mu \mathrm{A}$, respectively. Namely, the reduction current increased about fivefold (from AptDNA to hemin/AptDNA) and decreased about 1/2.5-fold (from hemin/AptDNA to hemin/AptDNA + histamine). Here, we can find the potential showing the largest change in current. In Fig. 4(c), the potential of $-0.023 \mathrm{~V}$ shows the largest current change. The current from AptDNA to hemin/AptDNA increased about 28-fold, and that from hemin/AptDNA to hemin/AptDNA + histamine decreased about $1 / 16$-fold. In this case, however, the changes should be considered only as a reference because the potential for measuring the current is close to the peak potential for the $\mathrm{H}_{2} \mathrm{O}_{2}$ reduction in the presence of residual $\mathrm{O}_{2}$. These data are summarized in Table 2.

Thus, we are able to observe the switching of electrochemical signals from $\mathrm{H}_{2} \mathrm{O}_{2}$ on the basis of the change in redox potential or current. In a previous study, we developed the probe DNA with a ferrocene moiety $(\mathrm{Fc})$ on one terminal and a $\beta$-cyclodextrin moiety $(\beta-\mathrm{CD})$ on the other terminal. ${ }^{(10)}$ This probe enabled the target recognition based on a "signal-on" architecture, increasing the redox current of Fc upon the sequence-specific recognition of target DNAs. ${ }^{(10)}$ Upon hybridization with the target, the dissociation of Fc, forming an inclusion complex with $\beta$-CD before hybridization, restores the electroactivity of $\mathrm{Fc}$ and negatively shifted the redox potential. Here, the current at a certain potential showed a fivefold increase upon hybridization. The prepared hemin/AptDNA was also revealed to switch electrochemical signals on or off in the absence or presence of histamine, respectively. This study is expected to contribute to the development of molecular probes with a switching function.

Table 2

Summary of reduction currents and their relative current intensities at the specific potentials for CVs for electrochemistry of AptDNA, hemin/AptDNA, and hemin/AptDNA + histamine in PBS containing $0.6 \mathrm{mM} \mathrm{H}_{2} \mathrm{O}_{2}$ [Fig. 4(b)] and $5 \mathrm{mM} \mathrm{H}_{2} \mathrm{O}_{2}$ [Fig. 4(c)].

\begin{tabular}{|c|c|c|c|c|c|c|}
\hline \multirow{3}{*}{$\begin{array}{l}\mathrm{H}_{2} \mathrm{O}_{2} \\
\text { Potential (V) }\end{array}$} & \multirow{2}{*}{\multicolumn{2}{|c|}{$\frac{0.6 \mathrm{mM} \text { [Fig. 4(b)] }}{-0.349}$}} & \multicolumn{4}{|c|}{$5 \mathrm{mM}$ [Fig. 4(c)] } \\
\hline & & & & 202 & & .023 \\
\hline & Current $(\mu \mathrm{A})$ & $\begin{array}{l}\text { Relative current } \\
\text { intensity }\end{array}$ & Current $(\mu \mathrm{A})$ & $\begin{array}{l}\text { Relative current } \\
\text { intensity }\end{array}$ & Current $(\mu \mathrm{A})$ & $\begin{array}{l}\text { Relative current } \\
\text { intensity }\end{array}$ \\
\hline AptDNA & -2.063 & 1 & -10.03 & 1 & -0.7547 & 1 \\
\hline hemin/AptDNA & -4.838 & 2 & -46.63 & 5 & -21.01 & 28 \\
\hline $\begin{array}{l}\text { hemin/AptDNA } \\
+ \text { histamine }\end{array}$ & -2.469 & $1 / 2$ & -18.44 & $1 / 2.5$ & -1.288 & $1 / 16$ \\
\hline
\end{tabular}




\section{Conclusions}

In this study, we demonstrated that the hemin/AptDNA aptazyme works as a mediator of reduction reaction between $\mathrm{H}_{2} \mathrm{O}_{2}$ and $\mathrm{ABTS}$ on the electrode surface, and that the activity of the mediator is switched on or off in the absence or presence of histamine as an inhibitor, respectively. This inhibitor-sensitive aptazyme can contribute to the development of molecular probes that enable molecular-recognition-based signal switching.

In contrast to natural enzymes that tend to be deactivated with short lifetimes, artificial-enzyme-based molecular probes have advantages and play important roles of contributing to the simplicity of sensing systems and the improvement of system handling. We are now designing new molecular probes on the basis of the thus developed aptazyme.

In hemin/AptDNA, the imidazole moiety is considered to coordinate the central metal Fe of hemin. For the development of molecular probes with a high switching effect, which means a greater difference in redox potential shift or absorption peak shift upon molecular recognition, further studies using other imidazole-containing inhibitors other than histamine are needed. We used histamine as a representative of imidazole-containing derivatives from the viewpoint of availability in this study to demonstrate a concept of artificial enzymes with a signal switching function. As a result, we were able to show the histamine-amenable switching effect on the reduction reaction of $\mathrm{H}_{2} \mathrm{O}_{2}$. We believe that we obtained relatively significant results to open a way to new molecular probes with a switching function.

\section{Acknowledgments}

We are grateful to Masaki Torimura of the Environmental Management Research Institute, National Institute of Advanced Industrial Science and Technology (AIST) for his help and useful advice regarding the use of artificial enzymes and comments on the manuscript. This work was partially supported by JSPS KAKENHI Grant Number JP19K05536 (awarded to HA).

\section{References}

1 E. D. Wagner and M. J. Plewa: J. Environ. Sci. 58 (2017) 64. https://doi.org/10.1016/j.jes.2017.04.021

2 B. Alexander-Dann, L. L. Pruteanu, E. Oerton, N. Sharma, I. Berindan-Neagoe, D. Módos, and A. Bender: Mol. Omics 14 (2018) 218. https://doi.org/10.1039/c8mo00042e

3 J. Wang, J. Chen, and S. Sen: J. Cell. Physiol. 231 (2016) 25. https://doi.org/10.1002/jep.25056

4 S. Kreth, M. Hübner, and L. C. Hinske: Anesth. Analg. 126 (2018) 670. https://doi.org/10.1213/ Ane.0000000000002444

5 A. Sassolas, B. D. Leca-Bouvier, and L. J. Blum: Chem. Rev. 108 (2008) 109. https://doi.org/10.1021/cr0684467

6 E. Paleček and M. Bartošík: Chem. Rev. 112 (2012) 3427. https://doi.org/10.1021/cr200303p

7 H. Aoki: Chem. Asian J. 10 (2015) 2560. https://doi.org/10.1002/asia.201500449

8 H. Aoki and H. Tao: Analyst 132 (2007) 784. https://doi.org/10.1039/b704214k

9 H. Aoki, M. Torimura, and T. Nakazato: Biosens. Bioelectron. 136 (2019) 76. https://doi.org/10.1016/ j.bios.2019.04.047

10 H. Aoki, A. Kitajima, and H. Tao: Supramol. Chem. 22 (2010) 455. https://doi.org/10.1080/10610278.2010.4860 34

11 C. Léger and P. Bertrand: Chem. Rev. 108 (2008) 2379. https://doi.org/10.1021/cr0680742

12 C. K. Prier and B. Kosjek: Curr. Opin. Chem. Biol. 49 (2019) 105. https://doi.org/10.1016/j.cbpa.2018.11.011

13 P. Travascio, P. K. Witting, A. G. Mauk, and D. Sen: J. Am. Chem. Soc. 123 (2001) 1337. https://doi. org $/ 10.1021 / \mathrm{ja} 0023534$ 
14 B. Li, H. Wei, and S. Dong: Strategy for use of smart routes to prepare label-free aptasensors for bioassay using different techniques. Aptamers in Bioanalysis, M. Mascini, Ed. (Wiley, New Jersey, 2009) p. 251.

15 M. Labib, E. H. Sargent, and S. O. Kelley: Chem. Rev. 116 (2016) 9001. https://doi.org/10.1021/acs. chemrev.6b00220

16 J. Ida, S. K. Chan, J. Glökler, Y. Y. Lim, Y. S. Choong, and T. S. Lim: Molecules 24 (2019) 1079. https://doi. org/10.3390/molecules24061079

17 Q. Loh and T. S. Lim: DNA G-Quadruplex as a Reporter System for Sensor Development: Nucleic Acids from Basic Aspects to Laboratory Tools, M. L. Larramendy and S. Soloneski, Eds. (IntechOpen, London, 2016), Chap. 7.

18 Y. Xiang and Y. Lu: Inorg. Chem. 53 (2014) 1925. https://doi.org/10.1021/ic4019103

19 X.-B. Zhang, R.-M. Kong, and Y. Lu: Annu. Rev. Anal. Chem. 4 (2011) 105. https://doi.org/10.1146/annurev. anchem.111808.073617

20 K. Sefah, J. A. Phillips, X. Xiong, L. Meng, D. van Simaeys, H. Chen, J. Martin, and W. Tan: Analyst 134 (2009) 1765. https://doi.org/10.1039/b905609m

21 P. Travascio, Y. Li, and D. Sen: Chem. Biol. 5 (1998) 505. https://doi.org/10.1016/s1074-5521(98)90006-0

22 S. E. Salamifar, S. Lee, and R. Y. Lai: Colloids Surf. B 123 (2014) 866. https://doi.org/10.1016/ j.colsurfb.2014.10.033

23 H. Aoki and H. Tao: Analyst 130 (2005) 1478. https://doi.org/10.1039/b507121f

24 H. Aoki and H. Tao: Anal. Sci. 24 (2008) 929. https://doi.org/10.2116/analsci.24.929

25 A. S. Wexler: Anal. Chem. 35 (1963) 1936. https://doi.org/DOI 10.1021/ac60205a045

26 X. Zhu, W. Zhang, H.-S. Xiao, J. Huang, and G. Li: Electrochim. Acta 53 (2008) 4407. https://doi.org/10.1016/ j.electacta.2008.01.042 